\title{
Eigenfaces Technique, an Improved Face Recognition Approach Using Neural Network
}

\author{
Mohd Ashraf ${ }^{1}$ and Md. Zair Hussain ${ }^{2}$ \\ ${ }^{1}$ Department of Computer Science and Engineering, ${ }^{2}$ Department of Information Technology, \\ ${ }^{1 \& 2}$ Maulana Azad National Urdu University, Hyderabad, Telangana, India \\ E-Mail: ashraf.saifee@gmail.com, mdzairhussain@gmail.com
}

\begin{abstract}
Image analysis and understanding, stands tall amongst all the technologies and face recognition is an eminent part of it. A face database is maintained as a logbook to identify an input face. This is accomplished by mere comparison amongst the face database. There are several face recognition techniques, of which, symmetry, Elastic Bunch Graph Matching (EBGM), and analytic-to-holistic recognition have been explored in this research paper. Other peculiar approaches like image based face recognition techniques like MLP, convolutional neural network, eigenfaces, associative neural networks, recirculation neural network and independent component analysis have been thoroughly discussed. Two vibrant face recognition databases, UMIST and ORL have proved to be extremely important in analyzing the results of face recognition. Eigen Face value approach has been anticipated with the associated analysis of results of face recognition. Another approach in face recognition is optimized multiperceptron, which will be acting as the reference to the optimized eigenfaces approach in this research paper, hence making this study more efficient through comparison.
\end{abstract}

Keywords: Biometry, Face Recognition, Principle Component Analysis, ORL Face Database, Multi-Layer Perceptron

\section{INTRODUCTION}

Human brains are biologically trained with this impeccable habit of recognizing faces since time immemorial. A beautiful instance of this is, a new born, recognizing its parents and relatives, without any training beforehand. This is a wow natural phenomenon. However, even with introductions in advanced level technologies, we are still unable to match up the natural, god-gifted, instincts. Success in this direction has become elusive. Several factors adds to contribute in the deviation from the accurate facial recognition, namely, large variation in orientation, facial appearances and expressions, head size and change in illumination. Usually, the most widely technique used is comparison of the input facial contours with the stored face database. The horizons of facial recognition are expanding every second with the advancements in technology. Applications where face recognition is explicitly used are personnel identification of credit cards, passport checks, entrance control, computer access control, crowd surveillance and ATM machines. Face recognition techniques are evolving every day to meet the quenching demands in various arenas. Today, the technology sound unfinished with this amazing evolution. Consequently, plethora of face recognition techniques has been developed and will still continue to develop, unless perfection is achieved.

The paper is divided in 5 peculiar sections. Section 2 presents the review of the previous work done in the area of face recognition. Section 3, discusses the details of eigenfaces based face recognition technique. Final analogies and results are portrayed in Section 4. While Section 5, concludes the findings.

\section{LITERATURE REVIEW}

In this section, we review the different face recognition techniques under different sub sections.

A. Geometric and Feature Based Approach: Facial contours and the geometric features play a significant role in face recognition, such as shape of eyes, mouth, nose, and chin and the exuberant relationship between them. Image pixels also add to the beauty of the facial recognition by introducing the feature based approaches. So, smaller the number of image pixels, better is the result. The feature based approaches have three major components: the definition of a feature set, the extraction of these features from a face image, and the recognition algorithm. A generalized symmetry operator is used in [2] to find the eyes and mouth in the face. The motivation stems from the almost symmetric nature of the face about a vertical line through the nose. Subsequent symmetries lie within features such as the eyes, nose and mouth. The symmetry operator locates points in the image corresponding to high values of a symmetry measure discussed in [2]. This method is independent of the scale or orientation. However, the search for symmetry points is computationally intensive.

B. Analytical-To-Holistic Based Approach: This is yet another approach, an analytic-to-holistic approach to face recognition based on a single frontal view [3] which yields exceptionally improvised results. The primitive step is to locate fifteen feature points on a face and analyze the face. The points are picked are based on the significance in representing face, and the reliability of extracting them automatically. The point distribution is quite dispersive in nature. Six feature points are located on the face boundary, four points for the eye corners, two for the mouth corners, two for the eyebrows and one for the nose. Dealing with 
face rotation and is effects is quite cumbersome, therefore, a head model is introduced to reduce this effect on face recognition. The point set for an input face is then compared with the point sets of the faces in a database by pointmatching. Faces similar to the input are chosen from the database and passed on for the second recognition process which measures the difference in context for the different facial features between the input and the database. Eyes, eyebrows window, nose window and mouth window are the major windows established for each face. 15 feature points on each face are spatial in the database. Three facial feature windows have a corresponding summation of gray-level intensity of each window.

C. Elastic Bunch Graph Matching (EBGM) Approach: In face recognition by elastic bunch graph matching (EBGM) [4], jets are the newly defines face features. Landmark locations are manually selected, from which jets are extracted. Jets are extracted from images and then collected in a data structure called a bunch graph. Every landmark on the face is associated with a bunch graph. The bunch graph has a node for every landmark on the face. The bunch graph serves as a database of landmark descriptions that can be used to locate landmarks in a new image. The EBGM algorithm computes the similarity of two images. To accomplish this task, the algorithm first finds landmark locations on the images that correspond to facial features such as the eyes, nose and mouth. Gabor wavelet convolutions are used at these points to describe the features of the landmark. Each image is represented by a face graph. A structure called a face graph is created where each node corresponds to a landmark, once the landmarks are located.

D. Neural Networks Based Approach to Face Recognition: Multilayer Perceptron (MLP) Neural Network is a good tool for classification purpose [5]. In [5], there is one hidden layer with 20 and 30 units in it. The number of units in the input layer is equal to the number of image pixels, 2576 (i.e.46x56). The number of output units is equal to the number of classes, i.e. 40; the number of persons in the ORL database. Each output unit has corresponding "own" class. A hyperbolic tangent is used as an activation function. A multilayer perceptron neural network [6] having one hidden layer with number of hidden units varying from 60 to80 was used which achieved a recognition rate from $94 \%$ to97\%. The input of the neural network is a set of discrete cosine transform coefficients. The first 30 coefficients from10304 are used.

A convolutional neural network for face recognition [7] has sophisticated architecture for image recognition. The input of such network is whole image. The system combines local image sampling, a self-organizing map (SOM) neural network and a convolutional neural network. The SOM provides a quantization of the image samples into a topological space where inputs that are nearby in the original space are also nearby in the output space, thereby providing dimensionality reduction and invariance to minor changes in the image sample, and the convolutional network provides for partial invariance to translation, rotation, scale, and deformation. The convolutional network extracts successively larger features in a hierarchical set of layers. Reported recognition rate is from $96 \%$ to $98.5 \%$.Autoassociative neural networks for face recognition [1]are special kinds of neural networks that are used to simulate associative processes in which the input patterns are associated with themselves. During training, patterns are presented to the network and weights are gradually adjusted in a way that the final pattern of connectivity matches all patterns being presented. One complete presentation of all patterns with which the network is trained is called one epoch; usually a network requires many such epochs to perform satisfactorily. The weights can therefore be seen as a distributed representation of the data. A face image cans be modeled using an auto associative memory. In this model, the face images are coded as a vector, $x_{k}$, whose elements give information on the gray levels of the corresponding pixels and these are the inputs of the system. Each element of the face vector $\mathrm{x}_{\mathrm{k}}$ is used as input to a cell of the auto associative memory. In this model, the number of cells in the auto associative memory is equal to the number of pixels in the face vector $\mathrm{x}_{\mathrm{k}}$. The output of a given cell for a given face is simply the sum of its inputs (the elements of the face vector, $\mathrm{x}_{\mathrm{k}}$ ) weighted by the connection strengths between itself and all of the other cells.

E. Neural Network Based Approach in Coordination with Principal Component Analysis: Face recognition using Eigenfaces, also called principal component analysis (PCA) [8] is fairly popular approach to face recognition. It encodes the most relevant information ina group of faces which will best distinguish them from one another. The approach transforms face images into a small set of characteristic feature images, called "Eigenfaces", which are the principal components of the initial training set of face images. Recognition is performed by projecting a new face image into the sub-space spanned by the eigenfaces (facespace) and then classifying the face by comparing its position in the face space with the position of known individuals. Each face image in the training set can be represented exactly in terms of a linear combination of the Eigenfaces and associated weights. A new face is recognized by comparing the feature weights needed to approximately reconstruct them with the weights associated with the known individuals.

Reconstruction and recognition of face images can also be performed using recirculation neural network (RNN) [9]. A recirculation neural network is based on multilayer perceptron, and it has one hidden layer. The number of units in the input and output layers is $n$ and equals to the number of pixels in the input image. The number of units in the hidden layer is $m$, where $m<<n$, and $m$ is equal to the number of principal components which is predefined. During the training process a neural network is learned to compress and reconstruct input images through the small number of hidden units, and the output of the hidden units is a compressed representation of the image. Recognition is 
performed by calculation of Euclidean distance between points; whose coordinates were the outputs of hidden units. The set of distances from the unknown image to all images from training set is calculated. An image with minimal distance to the unknown image is considered as recognized. Average recognition rate is about $92 \%$. The basis images found by PCA depend only on pair wise relationships between pixels in the image database. A generalization of PCA, Independent component analysis (ICA) [10] finds better basis images sensitive to the high-order relationships among pixels. The face images employed by them were a subset of the FERET face database. The data set contained images of 425 individuals. The images were cropped to $60 \mathrm{x}$ 50 pixels. The goal in this approach was to find a set of statistically independent basis images. A data matrix $\mathrm{X}$ was organized so that the images are in rows and the pixels are in columns, i.e., $X$ has 425 rows and 3000 columns, and each image has zero mean. ICA is performed on face images in the FERET database under two different architectures, one which treats the images as random variables and the pixels as outcomes, and a second which treats the pixels as random variables and the images as outcomes. The first architecture finds spatially local basis images for the faces. Under this architecture, ICA found a basis set of statistically independent images. The images in this basis set were sparse and localized in space, facial features. The second architecture as random variables and images as random trials. Under this architecture, the image coefficients were approximately independent, resulting in a factorial face code. Both ICA representations are superior to representations based on PCA for recognizing faces across days and changes in expression. A classifier that combines the two ICA representations gives the best performance.

A novel neural network architecture, which can recognize human faces with any view in a certain viewing angle range (from left 30 degrees to right 30 degrees out of plane rotation) is proposed in [11]. In this, view-specific Eigenface analysis is used as the front-end of the system to extract features, and the neural network ensemble is used for recognition. The approach is based on extending the Eigenface approach i.e. one Eigenface set is built for each view. Then the feature coefficients of each image are extracted in the corresponding Eigenspace. An ensemble neural network (consisting of two layers) is used as the classifier to perform the pose invariant face recognition. The first layer contains four view-specific neural networks, each of them is a conventional feed-forward network trained with the back propagation algorithm based on the training data of a specific view. Each network accepts the 20 dimensional Eigenface coefficients as the input vector, has 15 hidden units, and has 6 output units. The training data for each neural network contains 300 vectors, all of which are Eigenface coefficients calculated with the corresponding view. Among the 300 images, each of the 5 persons to be recognized contributes 40 images, and each of the 5 persons to be rejected contributes 20 images. The second layer is a combinational neural network trained on the output results of all the networks in the first layer. The output of the second layer network can not only tell the identity of the input image, but also tell the pose of the image, which is a bonus to the face recognition. The system achieves an average recognition ratio as high as $98.75 \%$.

\section{FACE RECOGNITION USING EIGENFACES}

The Eigenfaces method of face recognition is based on an information theory approach that decomposes face images into a small set of characteristic feature images called "eigenfaces", which may be thought of as the principal components of the initial training set of face images. Recognition is performed by projecting a new image into the subspace spanned by the eigenfaces ("face space") and then classifying the face by comparing its position in face space with the positions of known individuals.

A. EigenFace Approach: The Eigenfaces approach has been devised by Matthew A. Turk and Alex Pentland [9]. This approach to face recognition involves the following initialization operations.

1. Acquire an initial set of face images (the training set).

2. Calculate the eigenfaces from the training set, keeping only the $\mathrm{M}$ face images that correspond to the highest eigenvalues. These M images define the face space. As new faces are added, the eigenfaces can be updated or recalculated.

3. Calculate the corresponding distribution in $\mathrm{M}$ dimensional weight space for each known individual, by projecting their face images onto the "face space".

Having initialized the system, the following steps are then used to recognize new faces.

1. Calculate a set of weights based on the input image and the $\mathrm{M}$ eigenfaces by projecting the input image onto each of the eigenfaces.

2. Determine if the image is a face at all (whether known or unknown) by checking to see if the image is sufficiently close to "face space".

3. If it is a face, classify the weight pattern as either a known person or as unknown.

Now, we discuss the details of the development of a face recognition system based on eigenfaces. To implement the eigenfaces approach, we have taken the two different face databases namely UMIST face database and ORL face database.

B. Face Preprocessing and Eigen Analysis Steps: The various steps that are used in processing of the faces and in generating the eigenfaces and then using them for training the neural network are discussed as follows.

1. Cropping of the Face Images: When the face images are taken, then, the images also contain some background in addition to the actual face area. Cropping is the process of selecting the face area from the face images. Faces in the 
ORL database and UMIST database are reduced to 92 pixels in both heights and width as shown in Fig.1.

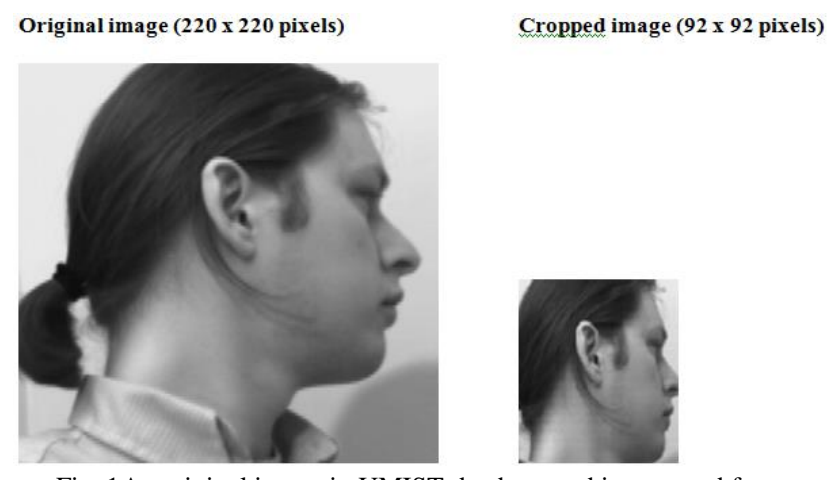

Fig. 1 An original image in UMIST database and its cropped form

2. Calculating the Mean Face: The mean face is calculated by taking the pixel-by-pixel average of all the faces in the database. Let the training set of $M$ face images be $\Gamma_{1}, \Gamma_{2}$, $\Gamma_{3} \ldots \Gamma_{M}$. The average face $\psi$ of the set is defined by:

$$
\Psi=\frac{1}{\mathrm{M}} \sum_{n=1}^{M} \Gamma_{n}
$$

During the calculations, the average of all the pixel values for the same point across the faces is taken, i.e., the values of the $(x, y)$ the pixel for all the faces are added and the sum is divided by the number of faces. The number of pixels in the mean image in both the ORL and UMIST databases is $92 \times 92=8464$. The total number of pixels is same in both the mean face and original faces.

In the ORL database, number of images is 250 (10 faces per person for 25 persons), so $M=250$. For the UMIST face database, 19 faces are taken for each person and number of persons considered is 15 so $M=285$. The mean faces for ORL and UMIST face databases are shown in fig. 2.

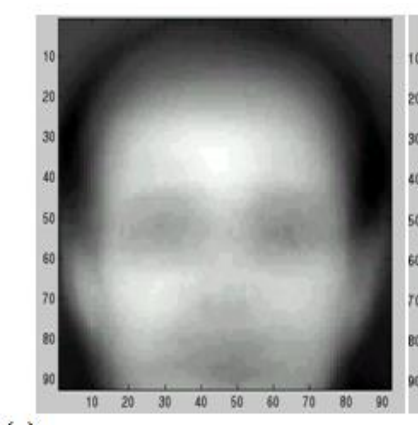

(a)

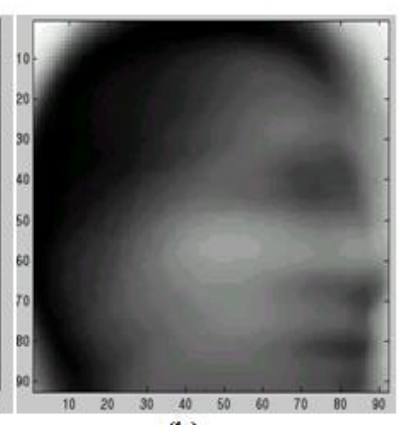

(b)
Fig. 2 (a) The ORL mean face (b) The UMIST mean face

3. Calculation of Difference Images: Once the mean image has been calculated, the mean image is subtracted from the individual face images one by one resulting in the difference images. Each face differs from the average by the vector $\Phi_{i}$ given by:

$$
\Phi_{i}=\Gamma_{i}-\Psi
$$

where $\Gamma_{i}$ is the $\mathrm{i}^{\text {th }}$ face image and $\Psi$ is the mean face image.This difference is calculated on a pixel-by-pixel basis, i.e., the value of the $(x, y)$ th pixel of the mean face is subtracted from the value of the $(x, y)$ th pixel of the original face image. Fig. 3 shows the original face image of the first person in ORL face database and also the difference image obtained after subtraction by the mean face image.

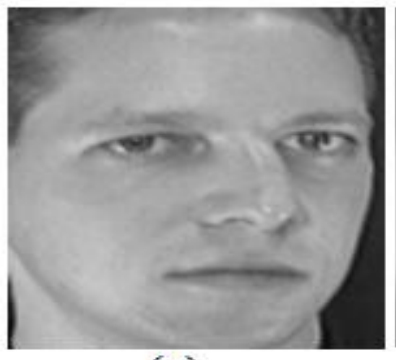

(a)

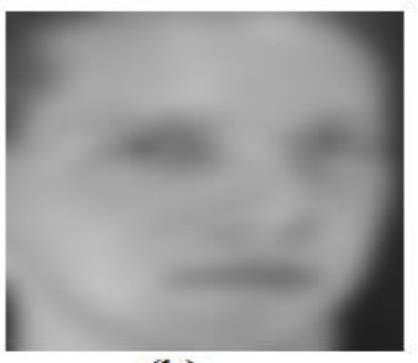

(b)
Fig. 3 (a) The original ORL database image (b) Corresponding difference image

The difference images capture the deviation of the original faces from the mean face. So the faces can be represented in terms of their deviations from the average face. An important property of the difference images is that the difference in the pixel values of the original and means image can be positive as well as negative, indicating positive or negative deviation from the mean image.

4. Calculation of the Covariance Matrix: After the calculation of the difference images for all the faces in the training set, we begin the calculation of the covariance matrix $\mathrm{C}$. The covariance matrix stores the difference images in a matrix form, thus capturing the deviations of the face images from the mean face. The covariance matrix represents the variations across the faces of the face database.

$$
C=\frac{1}{\mathrm{M}} \sum_{n=1}^{M} \Phi_{n} \Phi_{n}^{T}
$$

The above equation makes it clear that the covariance matrix is the element-by-element product between the difference matrix and transpose of the difference matrix calculated over all the $\mathrm{M}$ training faces. If a matrix $\mathrm{A}$ is considered such

$A=\left[\Phi_{1} \Phi_{2} \ldots \ldots \Phi_{M}\right]$. Then the covariance matrix can be represented as

$$
C=A A^{T}
$$

The Eigenvectors and Eigenvalues are defined for the covariance matrix. The matrix $C$, however, is $N^{2}$ by $N^{2}$ (where $N$ is the number of pixels in both width and height of each face image), and determining the $N^{2}$ eigenvectors and Eigenvalues is an intractable task for typical image sizes. We need a computationally feasible method to find these eigenvectors. For the ORL and UMIST databases the faces have been cropped to 92 pixels each in width and height, so the covariance matrix is of the order 8464 .

5. Determining the Eigenfaces: Rather than calculating the covariance matrix which is of the order of is $N^{2}$ by $\mathrm{N}^{2}$, we try to reduce the dimensionality of the matrix based on the approach of principal components. If the number of data points in the image is less than the dimension of the space, 
there will be only $M-1$, where $M$ is the number of faces in the training set, rather than $N^{2}$, meaningful eigenvectors. (The remaining eigenvectors will have associated eigenvalues of zero.) So we can solve for the $\mathrm{N}^{2}-$ dimensional eigenvectors by solving for the eigenvectors of an $M$ by $M$ matrix and then taking appropriate linear combinations of the face images $\Phi_{i}$. So the dimensionality of the covariance matrix can be reduced to the order of images in training set from the total number of pixels in the face image. We construct the $M$ by $M$ matrix $L=A^{T} A$, where

$$
L_{m n}=\Phi_{m}^{T} \Phi_{n}
$$

and find the $\mathrm{M}$ eigenvectors, $\mathrm{v}_{\mathrm{i}}$, of $L$, these vectors determine linear combinations of the $M$ training set face images to form the Eigenfaces $u_{i}$.

$$
u_{l}=\sum_{k=1}^{M} v_{l k} \Phi_{k}, l=1, \ldots \ldots, M
$$

The eigenfaces are just like the faces but are somewhat "ghostly" and are not as clear as the original faces. The eigenfaces are of the same size as the original faces, so they also have 92 pixels in width as well as in height.

For the ORL database, $M=250$ and total number of pixels $\left(N^{2}\right)=8464$ and for the UMIST database, $M=285$ and total number of pixels $\left(N^{2}\right)=8464$. So the numbers of eigenfaces that are needed to capture the variation of the ORL database are reduced from 8464 to 250 and for the UMIST face database only 285 eigenfaces are sufficient to represent the variations in the faces.

6. Sorting the Eigenfaces by Using Eigen Values: As a property of the eigenface, each of them has an eigenvalue associated with it. More important, eigenvectors with from the graph shown in Fig.4, where $\mathrm{x}$-axis represents number of eigenfaces and $y$-axis represents the corresponding eigenvalues, the number of eigenfaces having maximum eigenvalues is very less and the eigenvalues drop significantly after the top 50 eigenfaces.

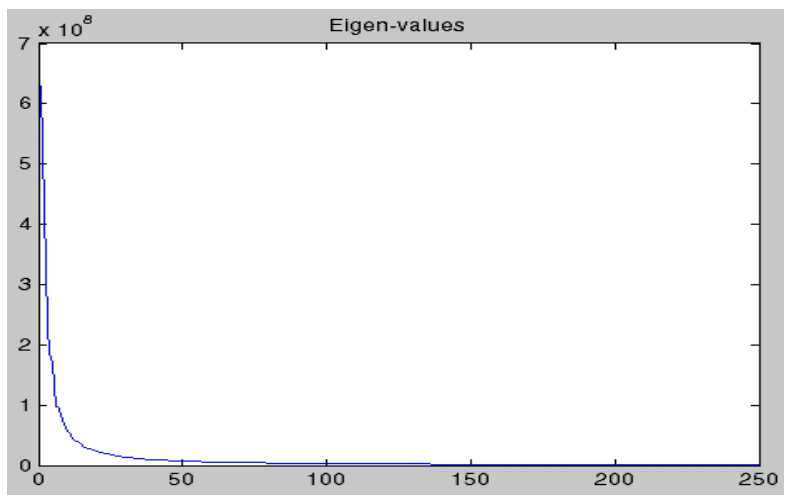

Fig. 4The variation in the Eigenvalues for ORL database

So, the faces are being represented using about 50 eigenfaces only. The eigenfaces with largest eigen values capture maximum variation of the faces while the eigenfaces with low eigen values are insignificant and do not represent much information about the faces. If we use the maximum number of eigenfaces then the faces can be most correctly reconstructed and recognized. For maximum accuracy, the number of eigenfaces should be equal to the number of images in the training set. In practice, a smaller $M^{\prime}$ is sufficient for identification. Therefore, the most significant $M^{\prime}$ eigenvectors of the $L$ matrix are chosen as those have the largest associated eigenvalues.

7. Face Reconstruction: After the calculation of the Eigenfaces, the original face images are reconstructed using the most significant eigenfaces. This is achieved by multiplying the eigenfaces with some multipliers (both negative and positive) and adding the products. In order to determinethe multipliers a new face image $(\Gamma)$ is transformed into its eigenface components by a simple operation,

$$
\omega_{k}=u_{k}^{T}(\Gamma-\Psi) \text { for } k=1, \ldots, M^{\prime} .
$$

The multipliers form a feature vector,

$$
\Omega^{T}=\left[\omega_{1}, \omega_{2} \ldots \ldots \omega_{M^{\prime}}\right]
$$

that describes the contribution of each eigenface in representing the input face image, treating the eigenfaces as a basis set for face images. Once the feature vector has been obtained, the original faces can be reconstructed by multiplying the Eigenfaces with the multipliers and adding up the products thus obtained. The number of multipliers will be same for all the faces and is equal to the number of eigenfaces that are sufficient for the reconstruction. In Fig.5, the original face and its reconstruction using the Eigenfaces is shown.

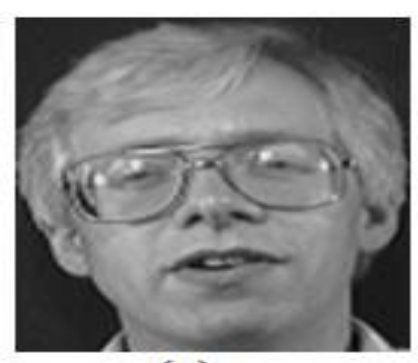

(a)

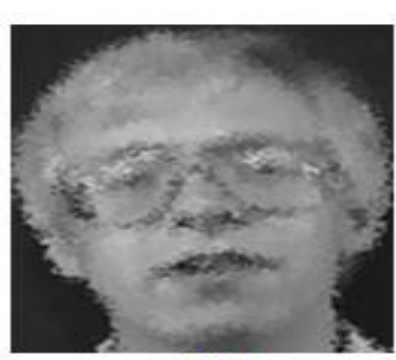

(b)
Fig. 5 (a) The original image(b) Its reconstruction using the multipliers

C. Generating the Input for the Neural Network: The neural network that is constructed for the face recognition task is supplied with inputs in the form of the multipliers that are generated. The original face images can be represented as the sum of products of the multipliers and the eigenfaces. So these multipliers can be used to differentiate the faces. The multipliers for all the faces of the same person are very much similar while they differ greatly from the multipliers of the faces of another person. The number of multipliers that are supplied to the neural network as the input is equal to the number of Eigenfaces that are sufficient to represent the variation of the faces. Now, we design an optimal multilayer neural network [15] for face recognition by specifying the parameter like learning rate, momentum, number of hidden layer and number of neurons in input layer, hidden layers and output layer. 


\section{EXPERIMENTS AND RESULTS}

In this section, we report here the results of our experiments, which were performed on the ORL and UMIST database. The UMIST university face database [12] consists of 564 images of 20 people, each covering a range of poses from profile to frontal views. Subjects cover a range of race/sex/appearance. The files are all in Portable Gray Map (PGM) format, 220 x220 pixels in 256 shades of grey. ORL database [13] contains a set of faces taken between April 1992 and April 1994 at Olivetti Research Laboratory (ORL) in Cambridge, UK. There are 10 different images are variations in facial expression (open/closed eyes, smiling/non-smiling), and facial details(glasses/no glasses) There is some variation in scale of up to about $10 \%$. The images are gray scale with a resolution of $92 \times 112$ pixels UMIST face database. The UMIST face database has a larger number of faces per person and also, the faces are of different poses. So the number of faces that can be used in training is more thus resulting in better recognition accuracy than that for the ORL database. The recognition accuracy is more than $95 \%$ as shown in Fig.6.

For the ORL faces, the recognition system performed very well. The recognition value for the correct person is around 0.9 and for all other persons it is very near to 0 (as shown in Fig.7). This clearly indicates that the face belongs to only the person with highest recognition value and not to the other persons.

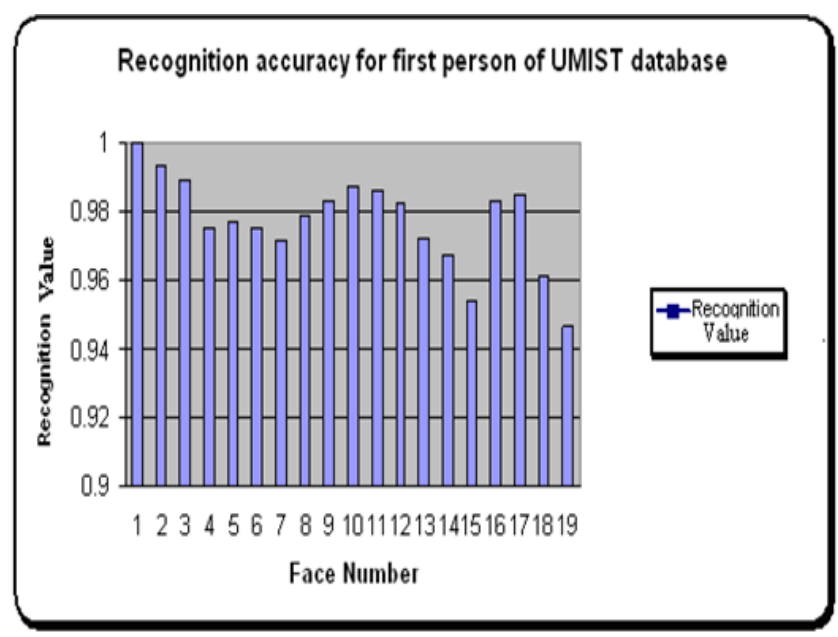

Fig. 6 Recognition value for first person of UMIST database

The number of Eigenfaces that are used in the reconstruction of the original faces plays an important role in determining the accuracy of the recognition system. Each original face is expressed as a sum of the products between the Eigenfaces and the multipliers. It was found that for the ORL face database, 50 Eigenfaces are sufficient as shown in Fig.8, resulting in an accuracy of more than $99.5 \%$ for the first person's faces. On the other hand, for the UMIST face database the number of Eigenfaces found to be sufficient is 403.

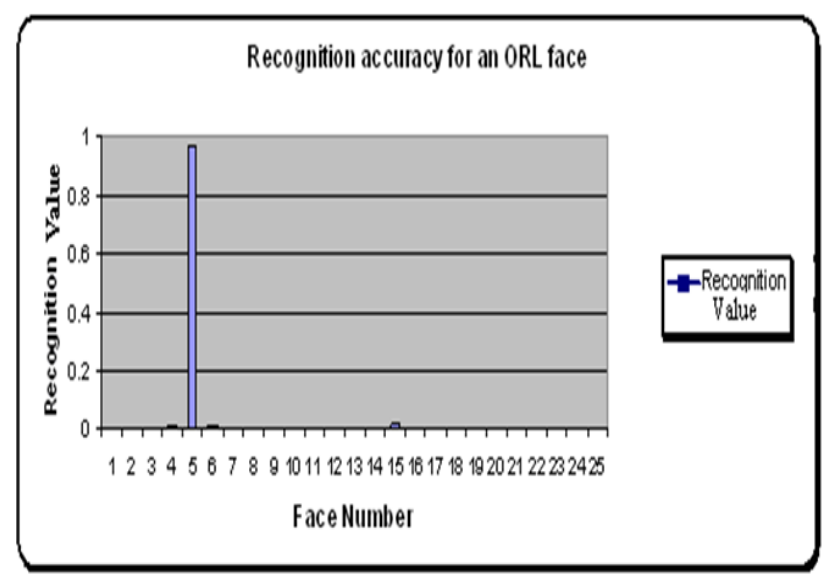

Fig. 7 Recognition accuracy for 25 persons when the face belongs to $5^{\text {th }}$ person

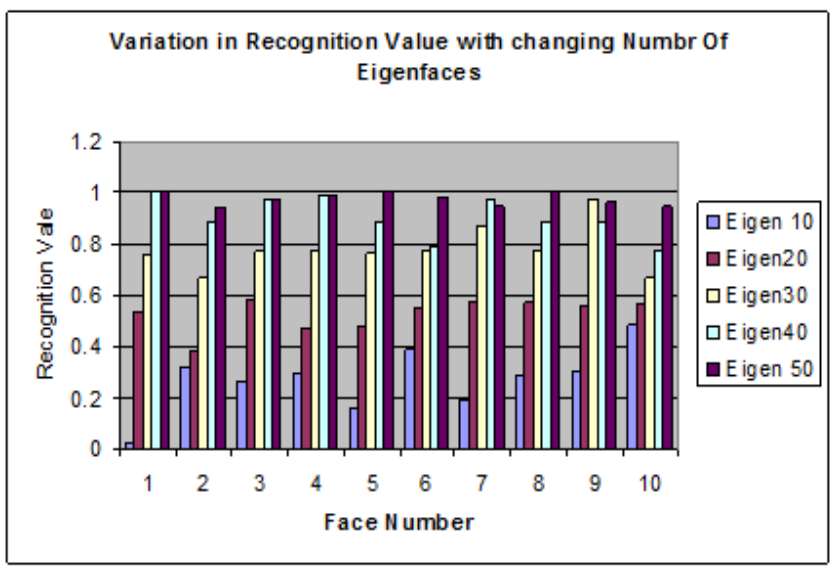

Fig. 8 Recognition value for first person of the ORL database with varying number of Eigenfaces

Now, we discuss the significance of results of the proposed method as shown in Table I in comparison to the results of the well-known face recognition methods as listed in Table II. The proposed method provides with the good accuracy with the less number of images per person in the training dataset, and also with the less number of design parameters for the neural network in comparison to the other techniques. In proposed method, number of input to the neural for the ORL face data base is 50 and the number of hidden layer is 2 and number of neurons units per hidden layer is 10 and 8 respectively. Similarly, for UMIST face database, number of input to the multi-layer perceptron (MLP) are 40 and the number of hidden layer is 1 and the number of neurons per layer is 11 respectively.

\section{CONCLUSION}

The various face recognition approaches varies in diversity with the diversity of the problems on face recognition. There are approaches like geometric and mathematical approaches but doesn't dwell where the Eigen face values and similar approaches persist. The approaches fail 
gradually when face recognition is to be done with new faces and not just the trained ones. If illuminations and pose change abruptly, the non-geometric approaches fail vehemently. The input space can also be reduced using PCA. PCA significantly reduces the computing complexity. Neural networks lend themselves for the task of face recognition by drawing the hyper-surfaces between the faces of the different persons. These methods however simplify the task of face recognition but the constraints are the variation in poses, illumination levels and facial features, thereby making the task quite difficult. This paper focuses on the optimized eigenfaces based approach, which uses the optimized multilayer perceptron for face recognition. This approach is found to be both proficient and efficient compared to the other classical approach.

TABLE I PROPOSED OPTIMIZE METHOD WITH PCA

\begin{tabular}{|l|c|c|c|c|c|}
\hline Type of NN & Input & $\begin{array}{c}\text { Number of } \\
\text { hidden layer }\end{array}$ & $\begin{array}{c}\text { Unit/ } \\
\text { hidden layer }\end{array}$ & Data Base & Accuracy \\
\hline MLP & 50 & 2 & 10,8 & ORL & $95 \%$ to $98 \%$ \\
\hline MLP & 40 & 1 & 11 & UMIST & $98.5 \%$ \\
\hline
\end{tabular}

TABLE II DIFFERENT METHOD RESULT

\begin{tabular}{|l|c|c|c|c|c|}
\hline \multicolumn{1}{|c|}{ Reference } & Type of NN & No. of input & $\begin{array}{c}\text { Hidden Unit/ } \\
\text { No of unit }\end{array}$ & Database & $\begin{array}{c}\text { Recognition } \\
\text { rate }\end{array}$ \\
\hline Z.Pen.et al.,[6] & MLP & Whole image & One (60 to 80) & ORL & $94 \%$ to97\% \\
\hline S.Lawrence et al.,[7] & ConvolutionNN & Whole image & & ORL & $96 \%$ to98.5\% \\
\hline S. Eickeleret al.,[14] & P-2D HMM & Whole image & & ORL & $98 \%$ to $100 \%$ \\
\hline V.Goloko, et al.,[9] & RNN & No of image pixel & $2(20$ to 30) & ORL & $97 \%$ \\
\hline Fu.Jie Huang et al.,[11] & Novel NN & No of image pixel & One, 15 to 30 & ORL & $98.75 \%$ \\
\hline
\end{tabular}

\section{REFERENCES}

[1] OnsenToygar and Adnan Acan, "Face Recognition using Auto associative Neural Networks: An experimental evaluation on the FERET database", In Proc. Of International XII Turkish symposium on artificial intelligence and neural networks, 2003.

[2] D. Reisfeld and Y. Yeshurun, "Robust Detection of Facial Features by Generalized Symmetry", In Proc. Of International Conference on Pattern Recognition, pp. 117- 120, 1992

[3] Kim-Man Lam and Hong Yan, "An Analytic-to-Holistic Approach for Face Recognition Based on a single Frontal view", IEEE Transactions on Pattern Analysis and Machine Intelligence, Vol. 20, No. 7, pp. 673-686, July 1998.

[4] Laurenz Wiskott, Jean-Marc Fellous, Norbert Kruger and Christoph von der Malsburg, "Face Recognition by Elastic Bunch Graph Matching", IEEE Transactions On Pattern Analysis And Machine Intelligence, Vol. 19, No. 7, pp. 775- 779, July 1997.

[5] Dmitry Bryliuk and Valery Starovoitov, "Access control by face recognition using neural networks and negative examples", In Proc. of The 2nd International Conference on Artificial Intelligence, Crimea, Ukraine, pp. 428-436, Sept.16-20, 2002.

[6] Z. Pan, A. G.Rust and H. Bolouri, "Image Redundancy Reduction for Neural Network Classification using Discrete Cosine Transforms", In Proc. of the International Joint Conference on Neural Networks, Vol. 3, pp. 149-154, 2000.

[7] S. Lawrence, C.L. Giles, A.C. Tsoi and A.D. Back, "Face Recognition: A Convolutional Neural Network Approach", IEEE
Transactions on Neural Networks, Special Issue on Neural Networks and Pattern Recognition, Vol. 8, No. 1, pp. 98-113, 1997.

[8] M. Turk and A. Pentland, "Eigenfaces for recognition", Journal of Cognitive Neuroscience, Vol. 3, pp. 72-86, 1991.

[9] Dmitry Bryliuk and Valery Starovoitov, "Application of recirculation neural network and principal component analysis for face recognition", In Proc. of the $2^{\text {nd }}$ International Conference on Neural Networks and Artificial Intelligence, Minsk, Belarus, pp. 136-142, Oct. 2-5, 2001.

[10] Marian Stewart Bartlett, Javier R. Movellan and Terrence J. Sejnowski, "Face Recognition by Independent Component Analysis", IEEE transactions on Neural Networks, Vol. 13, No. 6, pp. 14501464, Nov. 2002.

[11] Fu Jie Huang, Zhihua Zhou, Hong-Jiang Zhang and Tsuhan Chen, "Pose Invariant Face Recognition", In Proc. of the 4th IEEE International Conference on Automatic Face and Gesture Recognition, Grenoble, France, pp. 245-250, 2000.

[12] UMIST Face Database, [Online] Available at: http://images.ee.umist. .ac.uk/danny/database.html

[13] ORL Face Database [Online] Available at: www.cl.cam.ac.uk/resea rch/DTG/ attarchive: pub/data a/att_faces.tar.Z

[14] S. Eickeler, S. Muller, and G. Rigoll, "High performance face recognition using Pseudo 2-D Hidden Markov Models", In Proc. of European Control Conference (ECC),Aug.1999

[15] M. Ashraf and Rashid Ali, "Design of an Optimal Multilayer Neural Network for Eigenfaces Based Face Recognition," In the Proc. of IEEE sponsored International Conference on Recent Applications of Soft Computing in Engineering and Technology (RASIET-07), Alwar, India, pp. 253-263, 2007. 